\title{
Catatonía periódica o síndrome neuroléptico maligno: claves para un reto diagnóstico.
}

Periodic catatonia or neuroleptic malign syndrom: diagnostic clues.

\author{
Javier Sánchez García a , Livia de Rezende Borges a , Ignacio Vera López a . \\ ${ }^{a}$ Hospital Universitario de Getafe. Servicio de Psiquiatría. Getafe, Madrid, España.
}

Correspondencia: Dra.Livia de Rezende Borges (rezendelivia@hotmail.com)

Recibido: 11/12/2011; aceptado con modificaciones: 23/03/2012

\begin{abstract}
RESUMEN: Introducción: Los pacientes institucionalizados con enfermedad mental grave y alteraciones motoras plantean frecuentes dudas diagnósticas. La catatonía periódica se manifiesta por alteraciones motoras y signos orgánicos por lo que debe incluirse en el diagnóstico diferencial ante un cuadro sugestivo de síndrome neuroléptico maligno.

Caso clínico: Varón de 45 años institucionalizado por trastorno del desarrollo intelectual. Durante 3 primaversas consecutivas presenta insomnio, alteraciones conductuales persistentes, seguidas de catatonías acinéticas y síndrome neurovegetativo: rigidez, estereotipias, sudoración, taquicardia, febrícula y pérdida ponderal. Se le diagnostica de síndrome neuroléptico maligno retirándose los antipsicóticos. Reingresa sin solución de continuidad con importantes alteraciones conductuales que ceden tras reintroducción de los antipsicóticos. Emitido el diagnóstico de catatonía periódica se añade litio, sin recaídas hasta la fecha.

Discusión: Los cuadros psiquiátricos primarios graves, habituales antes de la introducción de los antipsicóticos todavía se observan en instituciones con difícil acceso a los servicios de salud mental. Debe contemplarse su diagnóstico en sujetos en los que concurren trastornos del neurodesarrollo con cambios bruscos de su funcionamiento.

PALABRAS CLAVE: catatonias psiquiátricas primarias, catatonia periódica, síndrome neuroléptico maligno, catatonia, pacientes psiquiátricos institucionalizados.
\end{abstract}

ABSTRACT: Introduction: Psychiatric institutionalized patients suffering severe learning disabilities and coincidental motor symptoms usually represent a challenge in their diagnostic and therapeutic processes. Periodic catatonia may produce neurovegetative and motor symptoms, the first being essential findings for an accurate diagnosis. Otherwise, these constellations of symptoms manifested in patients undergoing antipsychotic therapy might be misidentified as a neuroleptic malignant syndrome.

Case report: An institutionalized 45 year-old male with severe learning disability presented for 3 years seasonal behavioural changes: During the last winter weeks he experienced a purposeless excessive motor activity. Every springtime the patient associated insomnia and treatment-resistant hyperkinetic behavioural disturbances as a prelude of akinetic catatonia episodes with intercurrent neurovegetative syndrome (stereotyped movements, sweating, tachycardia, slight fever and severe weight loss). A diagnosis of malignant neuroleptic syndrome was made during his third episode zenith, and antipsychotic treatment removed; the patient must be readmitted to the hospital after severe behaviour disturbances, which remitted after antipsychotics reintroduction. The patient was subsequently diagnosed of periodical catatonia (bipolar disorder with catatonic features) and put on lithium carbonate-based therapy, presenting no relapses in the three next years.

Discussion: Some severe mental disorders characteristic from the pre-antipsychotic era can be still diagnosed affecting institutionalized patients commit in residential resources. So in spite of the tendency of considering psychotropic drugs and infections as the sole cause for this disorders, a primary psychiatric disturbance should also be considered in this kind of patients.

KEY WORDS: psychiatric catatonias, Periodic catatonia, neuroleptic malign syndrom, catatonia, institutionalized psychiatric patients. 


\section{Introducción:}

La atención de las personas con trastorno del desarrollo intelectual implica habilidades no siempre reconocidas curricularmente y en términos generales no constituye un aspecto monográfico de la formación psiquiátrica. Este hecho podría significar a la postre una discriminación generalizada de estos usuarios debido a las evidentes dificultades de manejo en los dispositivos convencionales. Por otra parte, cuanto más profundo es el déficit del sujeto más se activan actitudes nihilistas, asumiéndose en ocasiones que la incapacidad de estos pacientes para la interacción convencional exime al profesional en salud mental de su responsabilidad en el tratamiento. A su vez, la atención médica generalista también se topa con obstáculos estructurales y sociales, lo que paradójicamente no pocas veces deja al psiquiatra como único valedor para promover una apropiada atención somática de estos sujetos.

La catatonía es un síndrome psiquiátrico que cursa con una hipoactividad GABAérgica y dopaminergica y una hiperactividad glutamaérgica (1). Nosológicamente la psiquiatría moderna la clasifica según su posible etiología afectiva, esquizofrénica o médica (1), aunque sus hallazgos clínicos sean similares (2-3). La catatonía periódica (CP) (4), descrita en la psiquiatría clásica, se ha asociado tanto con la esquizofrenia como con la enfermedad afectiva bipolar (5-6). Es un cuadro poco frecuente $(4,7)$, tras la introducción de los neurolépticos a mediados del siglo pasado, no estando ubicado como tal en las actuales clasificaciones diagnósticas como la CIE-10 y el DSM IV-TR (8-9). Las CP tienen un amplio espectro clínico, pudiendo ser polimorfas y estacionales (6), presentarse como formas malignas hipertérmicas, con pérdida de peso, incontinencia de esfínteres, sudoración y taquicardia, así como alteración de la motilidad (6). Las alteraciones motrices descritas incluyen negativismo, rigidez, mutismo y estupor (5). El síndrome neuroléptico maligno (SNM) es un cuadro clínico grave (10), potencialmente mortal, causado por psicofármacos, que cursa con catatonía exógena y con alteraciones neurovegetativas y analíticas, como el aumento de la creatinin-fosfo-kinasa (CPK) y leucocitosis. A diferencia de la CP, el SNM ha aumentado su frecuencia con la extensión del uso de neurolépticos.

Los pacientes institucionalizados con enfermedad mental severa que inician un cuadro de catatonía o alteraciones motoras plantean frecuentes dudas diagnósticas, tanto desde el punto de vista psiquiátrico como del orgánico. Si además de alteraciones motoras concurren alteraciones somáticas que agraven la situación, el diagnóstico y el tratamiento se convierten en un problema hospitalario de manejo multidisciplinar. El objetivo de la exposición de caso clínico siguiente es plantear estas dificultades y recordar la posibilidad de que cuadros aparentemente "orgánicos" puedan tener un origen psiquiátrico primario. 
NOTAS CLÍNICAS

\section{Observación clínica:}

Varón de 45 años con encefalopatía hipóxica de origen perinatal, ingresado en una estructura residencial para personas con trastorno del desarrollo intelectual grave o profundo desde los 14 años de edad. Es incapaz de emitir lenguaje inteligible. Entre sus antecedentes personales más relevantes constan nódulos tiroideos normofuncionantes en seguimiento por Endocrinología. Tratamiento habitual: risperidona $9 \mathrm{mg} /$ día, lorazepam $3 \mathrm{mg} /$ día.

De forma estacional en 3 años consecutivos, presenta en primavera episodios regulares de las características que se referirán seguidamente y que requieren intensificación del tratamiento psicofarmacológico y diversas visitas al Servicio de Urgencias de su hospital de referencia. Así, entre los meses de mayo y julio comienza con insomnio que se sigue de alteraciones conductuales persistentes y de difícil manejo. Predomina a la exploración física una facies desencajada con una sucesión de muecas sin características distónicas, intensa sudoración y escaso parpadeo. Aparecen estereotipias en manos, manipulación y juego con heces. Reclama constantemente la presencia de cuidadores y comienza a aparecer una rigidez paulatina expresada en una deambulación robótica, aspecto de perplejidad con exoftalmos subjetivo y facies crispada. La frecuencia cardíaca se mantiene en 120 latidos por minuto, sin alteración en la tensión arterial. En 2 meses sufre una pérdida de peso de $12 \mathrm{~kg}$, a pesar de colocación de sonda nasogástrica por la que se administra dieta hipercalórica. Además desarrolla pérdida del control de esfínteres, dificultad para realizar movimientos bucofonatorios y consiguiente pérdida de capacidad para elaborar los sonidos que habían constituido durante toda su vida su única forma de comunicación. Presenta negativismo y rigidez como rasgos más prominentes en su la exploración de su motilidad. En resumen, durante tres años sufre catatonías acinéticas con síntomas neurovegetativos acompañantes.

Ante la intensa y rápida emaciación, a la que se sumó la presencia de febrícula en el último mes de su tercer episodio, se procedió a ingreso hospitalario bajo sospecha de malignización de sus nódulos tiroideso. Permanece ingresado durante 2 semanas en que se practican diversas pruebas complementarias: en su analítica de sangre destacaba un aumento de la CPK (1345 UI/L), aumento de proteína C reactiva $(76 \mathrm{mg} / \mathrm{L})$, Leucocitosis $(11.6103 / \mu \mathrm{L}$ con $80 \%$ de neutrofilos) y función tiroidea normal. Se realizó una radiografía de tórax que mostraba un infiltrado de características alveolares en lóbulo inferior izquierdo. La analítica de orina no mostraba alteraciones y la Tomografía axial computerizada toracoabdominal y craneal fueron normales. La punción lumbar y el análisis del líquido cefalorraquídeo no mostraron alteraciones. Es dado de alta tras punción tiroidea que descartaba malignidad en el bocio coloide quístico, desechándose esta causa como responsable del cuadro clínico. Se achaca pues la situación física del paciente al tratamiento 
psicofarmacológico, y se emite el diagnóstico de síndrome neuroléptico maligno y de neumonía aspirativa.

De acuerdo con ello se retira el tratamiento con risperidona, fármaco con el que cada año había presentado una respuesta conductual marcadamente favorable y se instaura tratamiento antibiótico. Tras tres días reaparecen alteraciones motoras y conductuales graves. Se inicia tratamiento con benzodiacepinas (Loracepam) y lamotrigina sin resultado, lo que ante la incapacidad de control conductual sin antipsicóticos motiva su reingreso.

En su segunda hospitalización se establece el diagnóstico probable de $\mathrm{CP}$ por los hallazgos clínicos compatibles (alteración motriz catatoniforme, taquicardia, febrícula y aumento de la CPK), que si bien similares a los del SNM tenían un curso estacional y se agravaban con la retirada de antipsicóticos. Se procedió a la introducción de olanzapina y periciazina, lográndose la adecuación conductual y de la motilidad. Ya en su centro residencial se introdujo litio, dado el carácter fásico del cuadro y la vinculación de las formas de catatonía periódica a los cuadros afectivos bipolares. En los tres últimos años no se han producido nuevas recaídas afectivas, alteraciones en la motilidad del paciente ni alteraciones neurovegetativas.

\section{Discusión:}

Es indiscutible que el síndrome neuroléptico maligno es una entidad a tener en cuenta en el diagnóstico diferencial de este paciente, debido a su semiología y a que el paciente recibía neurolépticos previamente al inicio del mismo (11). A pesar del juicio presuntivo de SNM, los datos clínicos y analíticos observados podían deberse a otras causas: La elevación de CPK podía explicarse por contracciones musculares violentas o la sujeción mecánica prolongada a la que fue sometido (tanto en su residencia como en el hospital). Tanto la leucocitosis como la febrícula persistente podían ser secundarias a la neumonía por aspiración. Había presentado taquicardia mantenida (ausente en los períodos de sueño) y no se observó alteración del nivel de conciencia. Cabe mencionar que el SNM se ha asociado al hipotiroidismo (10) y la catatonía periódica con el hipertiroidismo (5-6), lo que podría explicar la mejor la grave emaciación. En resumen, el aspecto decisivo en la presunción de SNM fue el antecedente de uso de antipsicóticos, tratamiento psicofarmacológico que, aún con ajustes, se seguía desde hacía años. Si bien la sospecha de SNM justifica inicialmente la retirada del antipsicótico, un incorrecto diagnóstico puede limitar en gran medida las opciones de tratamiento. Si bien el SNM y la CP son etiológicamente divergentes, se observa que comparten rasgos de 
su fenomenología, fisiopatología y de respuesta al tratamiento (12). Esta hipótesis considera ambos trastornos como una entidad única resultante de combinaciones variables de una hipoactividad del ácido gamma-aminobutírico en los receptores GABA-A, una hipoactividad de la dopamina en el receptor D2, una hiperactividad de serotonina en el receptor 5 - HT1A con hipoactividad en el receptor 5-HT 2A y una hipoactividad del glutamato en el receptor N-metil-D-aspartato (NMDA) (12). También se han descrito en la literatura casos de catatonía aguda tras SNM (13).

Un tercer diagnóstico diferencial sería la esquizofrenia catatónica. En la tabla 1 analizamos los datos clínicos distintitvos de estos cuadros. En los pacientes con retraso mental severo las infecciones son causa habitual de agitación psicomotriz y alteraciones conductuales persistentes, constituye así el primer diagnóstico a descartar. La incapacidad para comunicar síntomas y la clínica infecciosa compatible podría explicar su descompensación, aunque no sería compatible con el curso cíclico del cuadro inicial ni con el componente catatónico.

Tabla 1.

Diagnóstico diferencial entre Esquizofrenia catatónica, Catatonía Periódica y Síndrome Neuroléptico Maligno $(6,7,10,11)$.

\begin{tabular}{|c|c|c|c|}
\hline & $\begin{array}{l}\text { Síndrome Neuroléptico } \\
\text { Maligno }\end{array}$ & $\begin{array}{l}\text { Catatonía Periódica } \\
\text { (esquizofrenia no } \\
\text { sistemáticas) }\end{array}$ & $\begin{array}{l}\text { Esquizofrenia } \\
\text { catatónica (sistemática) }\end{array}$ \\
\hline Periodicidad & No & $\mathrm{Si}$ & No \\
\hline Curso & Agudo & Fasico & Insidioso, crónico \\
\hline $\begin{array}{l}\text { Síntomas } \\
\text { neurovegetativos }\end{array}$ & Sí & $\mathrm{Si}$ & Normalmente no \\
\hline Alteración motora & Posible & Sí & $\mathrm{Si}$ \\
\hline Alteración analítica & $\mathrm{Si}$ & $\mathrm{Si}$ & No necesariamente \\
\hline Hipotiroidismo & Descrita asociación & Descrita asociación & No relación descrita \\
\hline Clínica psicótica & $\mathrm{Si}$ & $\mathrm{Si}$ & $\mathrm{Si}$ \\
\hline $\begin{array}{l}\text { Estabilizadores de } \\
\text { ánimo }\end{array}$ & No & Mejoría clínica & No necesariamente \\
\hline Neurolépticos & Suspensión inmediata & Mejoría clínica & Mejoría clínica \\
\hline Tratamiento & Medidas de soporte & $\begin{array}{l}\text { TEC, benzodiacepinas } \\
\text { neurolépticos atípicos }\end{array}$ & $\begin{array}{l}\text { TEC, neurolépticos, } \\
\text { benzodiacepinas }\end{array}$ \\
\hline
\end{tabular}

TEC: Terapia electroconvulsiva. 
Mientras el tratamiento del SNM sería el soporte y la retirada inmediata de los neurolépticos, en la CP serían las benzodiacepinas y la Terapia Electroconvulsiva (14), existiendo series de casos tratados con olanzapina (7), risperidona (11) o con mirtazapina (14). Los estabilizadores del ánimo como el litio podrían ser útiles, como en el caso de nuestro paciente, que permanece estable desde hace 3 años.

La psiquiatría clásica describió numerosos cuadros que, bien por su baja frecuencia o por las clasificaciones vigentes, impiden que sean considerados en el diagnóstico habitual. La presunta erradicación de cuadros graves tras la aparición de la medicación antipsicótica no parece aplicable a diversos contextos tanto en nuestro ámbito como en otros países, cuando se trata de personas con dificultades de acceso a atención especializada. A pesar de la integración de la atención psiquiátrica en los hospitales generales, continúa siendo preocupante la tendencia de los distintos especialistas médicos a considerar los tratamientos psicofarmacológicos más como causantes de problemas que como soluciones a los mismos, con inadecuadas actuaciones cuyo origen debe seguir estableciéndose.

BIBLIOGRAFÍA:

(1) Carroll BT, Kirhart R, Ahuja N, Soovere I, Lauterbach EC, Dhossche D, et al. A new conceptual understanding of catatonia and a new rating scale. Psychiatry (Edgmont) 2008; 5: 42-50.

(2) Carroll BT, Kennedy JC, Goforth HW. Catatonic signs in medical and psychiatric catatonias. CNS Spectr 2000 Jul;5(7): 66-69.

(3) Parellada E, Araúxo A, Bernardo M. Diagnostico diferencial de un síndrome catatonico: a propósito de un caso. Med Clin (Barc) 1991; 20: 597.

(4) Carroll B, Yoho SD, Bottoms JM. Periodic catatonia. Ann Clin Psychiatry 2011 2011; 23: $150-151$.

(5) Hardwick SW, Strokes AB. Metabolic Investigations in Periodic Catatonia. Proc R Soc Med 1941; 24: 733-756.

(6) Monchablon A. Catatonía Periódica (Catatonías endogógenas Agudas). Alcmeon 1991; 4: 566-586.

(7) Guzman CS, Myung VH, Wang YP. Treatment of periodic catatonia with olanzapine: a case report. Rev Bras Psiquiatr 2007; 29: 380.

(8) DSM-IV-TR Manual diagnóstico y estadístico de los trastornos mentales. Barcelona: Masson, 2002.

(9) Organización Mundial de la Salud. CIE-10. Décima Clasificación Internacional de las Enfermedades. Trastornos mentales y del comportamiento. Madrid: Meditor, 1992.

(10) Monchablon A. Síndrome Neuroléptico Maligno (Catatonía Neuroléptica Maligna)- su tratamiento con TEC. Alcmeon 1990; 1: 5-38.

(11) Duggal HS, Gandotra G. Risperidone treatment of periodic catatonia. Can J Psychiatry 2005; 50: 241-242. 
(12) Carroll BT. The universal field hypothesis of catatonia and neuroleptic malignant syndrome. CNS Spectr 2000; 5: 26-33.

(13) Benjelloun G, Jehel L, Abgrall G, Pelissolo A, Allilaire J. Acute catatonic syndrome after neuroleptic malignant syndrome. Encephale 2005; 31: 705-9.

(14) Yeh YW, Chen CY, Kuo SC, Wang TY, Wang HY, Chen CL. Mirtazapine treatment of periodic catatonia in organic mental disorder: a case report. Prog Neuropsychopharmacol Biol Psychiatry 2010; 16: 553-554. 